\title{
Use of real-time ultrasound imaging for biofeedback of diaphragm motion during normal breathing in healthy subjects
}

\author{
Ji-Eun Cho ${ }^{a}$, Dal-Yeon Hwang ${ }^{b}$, Joohee Hahn ${ }^{a}$, Wan-Hee Lee ${ }^{c}$ \\ ${ }^{a}$ Department of Physical Therapy, Graduate School, Sahmyook University, Seoul, Republic of Korea \\ ${ }^{b}$ Department of Rehabilitation Medicine, Myongji Choonhye Rehabilitation Hospital, Seoul, Republic of Korea \\ 'Department of Physical Therapy, College of Health Science, Sahmyook University, Seoul, Republic of Korea
}

Objective: To determine if the provision of visual biofeedback using real-time rehabilitative ultrasound imaging (RUSI) enhances the acquisition and retention of diaphragm muscle recruitment during exercise.

Design: Two group pretest posttest design.

Methods: Thirty healthy subjects were randomly assigned to the verbal feedback group (VG, $\mathrm{n}=15)$ or the visual and verbal feedback group (VVG, $\mathrm{n}=15$ ). The VG performed breathing exercises 10 times with verbal feedback, and the VVG also performed breathing exercises 10 times with verbal feedback and visual feedback with the use of RUSI to measure changes in diaphragm thickness (DT). For DT, the mid-axillary lines between ribs 8 and 9 on both sides were measured in standing, and then the chest wall was perpendicularly illuminated using a linear transducer with the patients in supine to observe the region between rib 8 and 9 and to obtain 2-dimensional images. DT was measured as the distance between the two parallel lines that appeared bright in the middle of the pleura and the peritoneum. After one week, three repetitions (follow-up session) were performed to confirm retention effects. Intra- and between- group percent changes in diaphragm muscle thickness were assessed.

Results: In the VVG, the intervention value had a medium effect size compared to the baseline value, but the follow-up value decreased to a small effect size. In the between-group comparisons, during the intervention session, the VVG showed no significant effect on percent change of DT but had a medium effect size compared to the VG ( $p=0.050$, Cohen's d $=0.764)$. During the follow-up session, retention effect did not persist ( $p=0.311$, Cohen's $\mathrm{d}=0.381$ ).

Conclusions: RUSI can be used to provide visual biofeedback and improve performance and retention in the ability to activate the diaphragm muscle in healthy subjects. Future research needs to establish a protocol for respiratory intervention to maintain the effect of diaphragmatic breathing training using RUSI with visual feedback.

Key Words: Biofeedback, Diaphragm, Respiratory muscles, Ultrasonography

\section{Introduction}

The diaphragm is well known as the principal muscle of respiration. The dome-shaped musculotendinous structure contracts during inspiration, flattening the pleural cavity, and expanding the lungs. When diaphragmatic function is impaired, the accessory muscles must assume this role but they are much less efficient, resulting in shortness of breath with exertion in patients with diaphragmatic dysfunction [1]. The diaphragm is important for mechanical stability in the upper part of the trunk [2]. Contraction of the diaphragm increases intra-abdominal pressure, working synergistically with the pelvic floor and abdominal muscles to increase spinal stiffness and stability $[3,4]$. The diaphragm may also increase spinal stability directly via the attachment of its crura to the lumbar vertebrae [5]. Previous studies have shown

Received: 21 March, 2018 Revised: 18 April, 2018 Accepted: 20 April, 2018

Corresponding author: Wan-Hee Lee (ORCID https://orcid.org/0000-0001-8030-4853)

Department of Physical Therapy, College of Health Science, Sahmyook University, 815 Hwarang-ro, Nowon-gu, Seoul 01795, Republic of Korea Tel: 82-2-3399-1633 Fax: 82-2-3399-1639 E-mail: whlee@syu.ac.kr

(c) This is an Open-Access article distributed under the terms of the Creative Commons Attribution Non-Commercial License (http://creativecommons.org/licenses/ by-nc/4.0) which permits unrestricted non-commercial use, distribution, and reproduction in any medium, provided the original work is properly cited.

Copyright $@ 2018$ Korean Academy of Physical Therapy Rehabilitation Science 
that, compared to healthy subjects, patients with chronic low back pain (LBP) and sacroiliac joint pain have abnormal diaphragm movement and/or position during tidal breathing with postural tasks [6,7]. Kolar et al. [6] found that patients with LBP had less motion (excursion) of the diaphragm with dynamic loading of the limbs during normal breathing. Cho et al. [8] also reported diaphragmatic weakness in hemiplegic patients with stroke, which affected thoraco-abdominal motion and resulted in ineffective trunk movement.

Despite the importance of the diaphragm for respiration and spinal stability, knowledge of training methods for diaphragmatic motion is limited. Diaphragmatic breathing exercises are commonly performed to train the diaphragm by breathing through the nose, filling the abdomen with air, and slowly exhaling through pursed lips [9]. During this, therapists may offer verbal or tactile feedback to provide resistance to the abdomen. However, it is difficult to determine if diaphragmatic breathing exercises are successfully trained. Furthermore, patients with difficulty in voluntary muscle contraction due to problems, such as muscle weakness, reduced sensation, and poor proprioception (e.g., stroke patients) are barely trained in diaphragm breathing. Therefore, it is necessary to provide feedback via new methods to train muscles effectively.

Rehabilitation ultrasound images (RUSI) reliably record changes in muscle thickness, fiber length, pennation angle, and cross-sectional area [10]. RUSI, which can provide visual feedback by showing changes in muscle thickness in real time, has also been used for neuromuscular retraining of the transverse abdominis or multifidus muscles, which are difficult to contract voluntarily [7,11]. Research has demonstrated the effectiveness of augmenting clinical instruction with visual feedback via RUSI for enhancing performance of isolated contraction of the transverse abdominis and lumbar multifidus muscles $[11,12]$. RUSI was used as a form of feedback to enhance the motor learning process for isolated recruitment of the deep muscle of the spine [11,13]. Although the diaphragm plays an important role in trunk stability with these trunk muscles, there are few studies suggesting effective ways to train the diaphragm. Among these, Kim et al. [14] was the only one that presented audiovisual biofeedback using magnetic resonance imaging to improve diaphragmatic motion. In addition, to date, there are no studies that have explored the effects of visual biofeedback using RUSI on learning to recruit the diaphragm muscle and its retention effects. Therefore, the purpose of this study is to investigate the motor learning effect of visual biofeedback us- ing RUSI in diaphragm muscle recruitment. Our hypothesis is that visual biofeedback using RUSI will be more effective in inducing changes in diaphragm muscle thickness compared to conventional breathing exercises. We also hypothesize that these changes will cause permanent changes in motor learning of the diaphragm, also known as retention effects.

\section{Methods}

\section{Participants}

A total of 30 healthy normal adult volunteers aged 24 to 33 years were studied. Subjects of both sexes were included and randomly allocated to the verbal feedback group (VG) or the visual and verbal feedback group (VVG) based on the selection of either number 1 or 2 from a sealed envelope. The VG consisted of 9 females and 6 males (mean \pm standard deviation [SD], 26.13 \pm 2.74 years) and the VVG consisted of 8 females and 7 males (mean \pm SD, 25.93 \pm 2.49 years). Exclusion criteria were previous lumbar or rib injury or surgery that altered the diaphragm muscles, known neuromuscular or joint disease, prior experience with biofeedback using ultrasound imaging, and prior training in cognitive activation of the diaphragm muscle. All subjects provided their informed consent. The study was approved by the Medical Research Ethics Committee at Sahmyook University in Seoul (IRB No. 2-1040781-AB-N-01-2017070HR).

\section{Procedures}

We conducted a randomized controlled trial in which participants were equally randomized to either the VG or VVG. Participants were allocated using a computer-generated block-randomization sequence using numbers contained in a sealed envelope. Participants were blinded to allocation, and end-point assessments were undertaken by an observer blinded to allocation. All the allocations were performed by a physical therapist who was not involved in the study. Prior to testing in the acquisition phase, all subjects received the same initial explanation relating to the diaphragm muscle. It was explained to the subjects that the contraction would be detected and measured using the ultrasound imaging equipment, and that they would have 5 seconds to contract the diaphragm muscle and hold the contraction. At the end of the 5 -second period, the image was saved on the screen, and the measurement of the resultant increase in thickness was performed. Each subject performed a total of 10 contractions (intervention session), as it was understood that 
more repetitions may induce fatigue. Twenty seconds of rest were provided between contractions. All subjects received verbal feedback on the increase in muscle thickness measured in millimeters that occurred with contraction of the diaphragm. In addition to the provision of verbal feedback, subjects in the VVG received biofeedback in the form of visual observation from the RUSI of the muscle contraction as it occurred (verbal plus visual feedback). Subjects were positioned in supine position and visual feedback from the RUSI was achieved by use of a personal computer monitor (Figure 1). Ultrasonographic measurements of all subjects during training were performed by one assessor with an ultrasound experience of more than 5 years.

After completing the 10 trials in the acquisition phase, all subjects were asked to return in one week for follow-up assessments to confirm the retention effects. The assessor who performed the follow-up assessments was blinded to group allocation. A one-week period between the intervention session and the follow-up session was chosen as representative of the gap period that commonly exists between treatment sessions in physical therapy practice. Subjects were instructed not to practice the contraction during the one-week period. The second session involved three more trials of the same contraction; however, the subjects were not provided with any biofeedback on their performance nor the results, for all 3 trials.

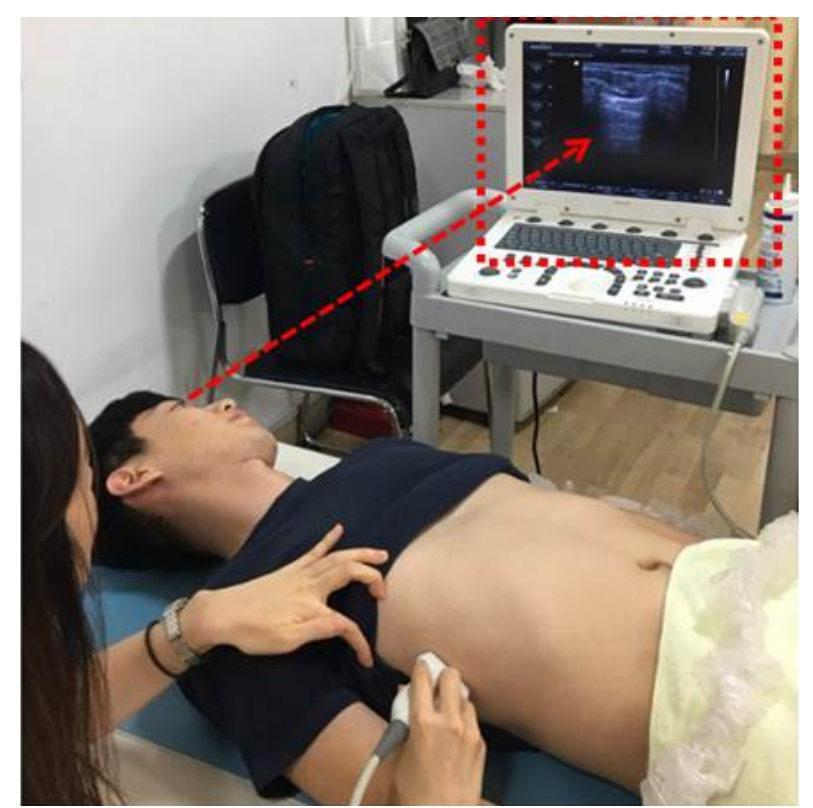

Figure 1. Biofeedback training in the form of visual observation of the ultrasound image of the diaphragm muscle contraction.
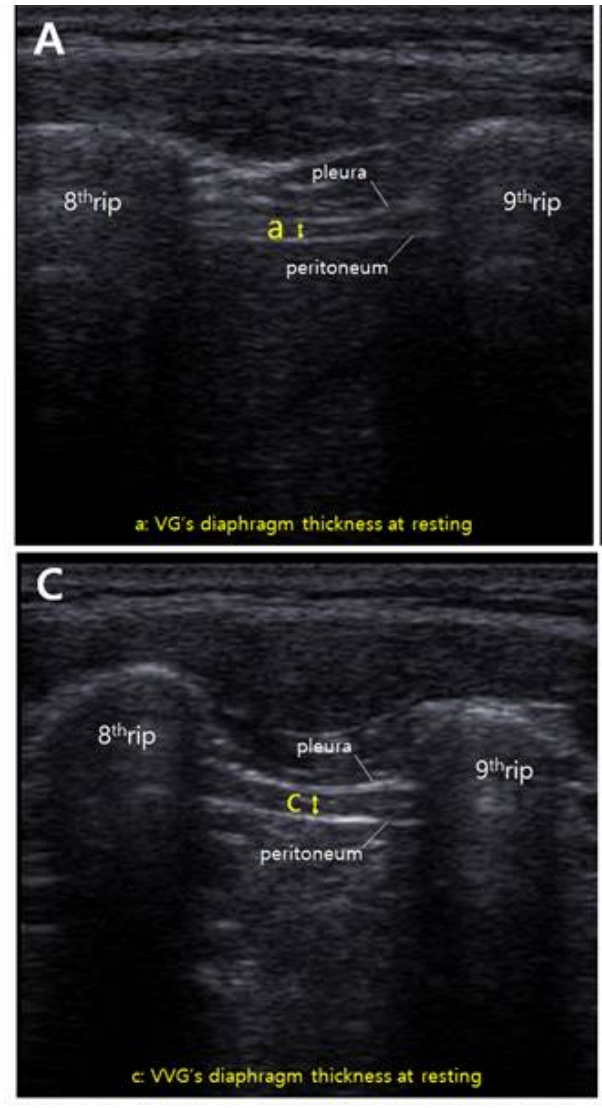

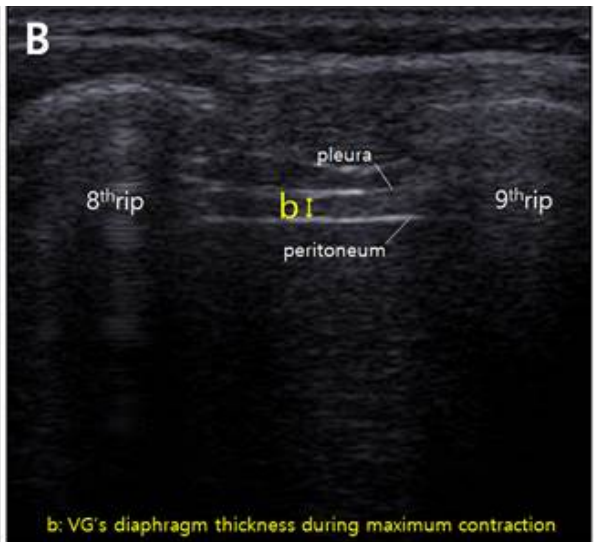

D

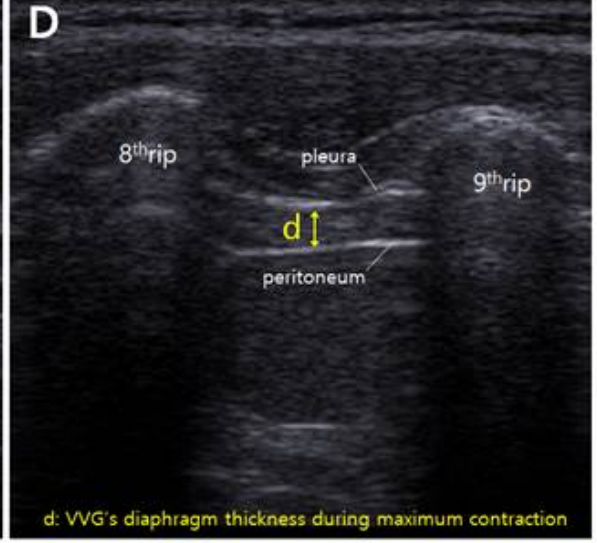

Figure 2. Comparison in the VG (A, $\mathrm{B})$ and the verbal and visual feedback group (C, D) ultrasound measurements of diaphragm thickness at rest and during maximal contraction using real-time rehabilitative ultrasound image. VG: verbal feedback group, VVG: visual and verbal feedback group. 


\section{Outcome measures}

\section{Diaphragm thickness}

A real-time B-mode ultrasound imaging system (MYSONO U5; Samsung Medicine, Seoul, Korea) with a 5.0-14.0 MHz linear transducer was used for examination of the diaphragm muscle thickness at rest and during maximum voluntary contraction. For measurement of diaphragm thickness (DT), the mid-axillary lines between ribs 8 and 9 on both sides were examined for all patients in a standing posture, and then the chest wall was perpendicularly illuminated using a linear transducer with the patients in supine position to observe the region between rib 8 and rib 9 and to obtain 2-dimensional images (Figure 2). The DT was measured as the distance between the two parallel lines that appeared bright in the middle of the pleura and in the middle peritoneum (Figure 2) [8].

\section{Statistical analysis}

For the diaphragm muscle contraction measures, the amount of increase in thickness on contraction was expressed as a percentage of the resting muscle thickness. The formula used for each of the 13 trials performed (10 intervention and 3 follow-up) was: percentage increase $=[$ (contracted thickness - resting thickness) $/$ (resting thickness) $] \times 100 \%$. Results were presented as means \pm SDs. Prior to all analyses, the normality of the data was assessed with a one-sample KolmogorovSmirnov test. Comparisons of the changes between the groups were performed using independent t-tests. Baseline, intervention, and follow-up data were compared within groups using a paired t-test. For all tests, statistical significance was set at $\alpha=0.05$. All statistical analyses were performed using IBM SPSS Statistics ver. 21.0 software (IBM Co., Armonk, NY, USA).

Cohen's d was applied for effect size calculations using the difference between two means divided by the pooled SDs. Firstly, changes in diaphragm percentages were analyzed for comparison between the two groups. Secondly, intra-group comparisons were analyzed for baseline versus intervention, and intervention versus follow-up results. Effect sizes ranging from 0.2 to 0.5 , from 0.5 to 0.8 , and from 0.8 to infinity were defined as small, medium, and large, respectively [15].

\section{Results}

Thirty participants were recruited for the study (15 in the VG and 15 in the VVG). All participants completed all tests

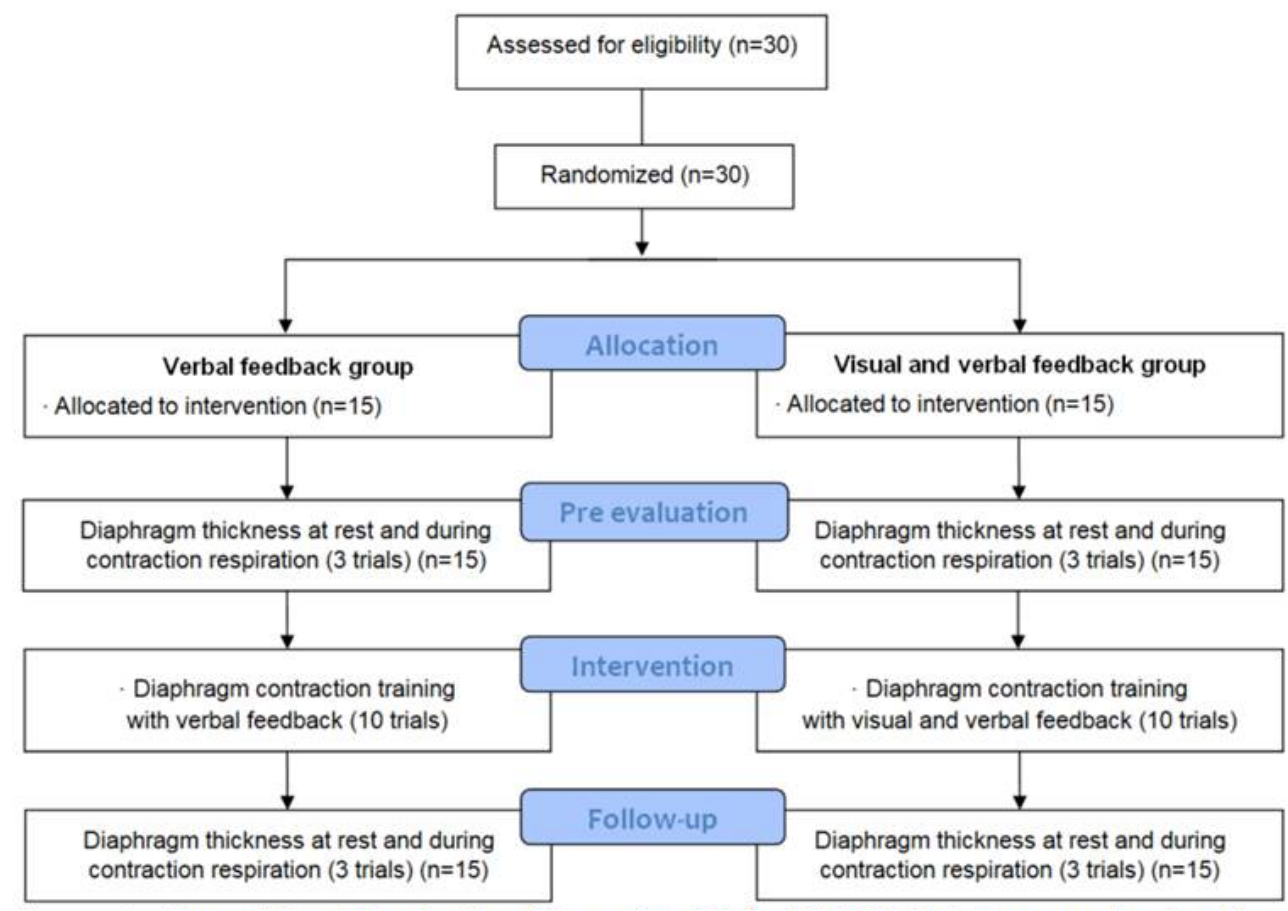

Figure 3. Consolidated Standards of Reporting Trials diagram showing flow of participants through each stage of this randomized trial. Participants were screened and randomly assigned to groups. 
and training (Figure 3). Participant characteristics for both groups are listed in Table 1. There were no significant differences between the groups with regards to base characteristics and baseline values at the beginning of the study $(p>0.05)$.

Following 10 intervention trials, the VVG showed no significant improvement in the percent increase of DT $(p=0.102)$, but showed a medium effect size (Cohen's $\mathrm{d}=0.502$ ) compared to the baseline values. The VG also showed no significant improvement in percent increase of DT $(p=0.248)$, and showed a small effect size (Cohen's $\mathrm{d}=0.273$ ). After 3 follow-up trials, the VVG showed no significant improvement in the percent increase of DT $(p=0.339)$, and showed a small effect size (Cohen's $\mathrm{d}=0.261$ ) compared to their intervention trial values. The VG also showed no significant improvement in the percent increase of DT ( $p=0.743)$, and showed a small effect size (Cohen's d=0.072) (Table 2).

In the between-group comparisons, during the 10 intervention trials, VVG showed no significant effect on the percent change of DT but showed a medium effect size compared to VG ( $p=0.050$, Cohen's $\mathrm{d}=0.764)$. During 3 follow-up trials, VVG showed a small effect size compared with that of the VG, and there was no significant effect in the VVG on the percent change of DT compared with that of VG

Table 1. Demographic characteristics

$(\mathrm{N}=30)$

\begin{tabular}{lccc}
\hline \multicolumn{1}{c}{ Characteristic } & VG $(\mathrm{n}=15)$ & VVG $(\mathrm{n}=15)$ & $\mathrm{t}(p)$ \\
\hline Sex (female/male) & $9 / 6$ & $8 / 7$ & $0.357(0.724)$ \\
Age (y) & $26.13(2.74)$ & $25.93(2.49)$ & $0.209(0.836)$ \\
Height $(\mathrm{m})$ & $166.86(8.68)$ & $165.86(7.45)$ & $0.338(0.738)$ \\
Weight $(\mathrm{kg})$ & $64.86(14.09)$ & $62.66(13.88)$ & $0.673(0.670)$ \\
\hline
\end{tabular}

Values are presented as number only or mean (SD).

VG: verbal feedback group, VVG: visual and verbal feedback group. $(p=0.311$, Cohen's $\mathrm{d}=0.381)$ (Table 2).

\section{Discussion}

The purpose of this study was to determine if visual biofeedback using RUSI is effective for DT changes during training of the diaphragm muscles and if this training has a lasting effect. The results of this study indicate that the visual biofeedback group with verbal feedback showed no significant changes in diaphragm percent change at rest and during maximal contraction compared with the verbal feedback only group. However, the results of the effect size analysis support that visual biofeedback is effective in retraining diaphragm movements. This group did not maintain the retention effect.

The diaphragm is well known as the principal muscle of respiration and contributes to the mechanical stabilization of the trunk [2]. Contraction of the diaphragm increases intra-abdominal pressure, working synergistically with the pelvic floor and abdominal muscles to increase trunk stability [3]. There is evidence that the diaphragm contributes to spinal stability, and previous studies have established a link between diaphragm dysfunction and LBP and sacroiliac pain $[6,7]$. Subjects with LBP have been shown to have decreased proprioception [16], which affects their ability to provide and process internal feedback, and therefore, augmented biofeedback may be indicated. Other evidence suggests that a 6-week breathing training for stroke patients with asymmetric weakening of the diaphragm improves their balancing ability [17] and gait endurance [8], which precede postural control. This explains the importance of the diaphragm as a stabilizer of the trunk, which indicates that a training method reflecting biofeedback targeting only the di-

Table 2. Results of the percent increase of diaphragm thickness

$(\mathrm{N}=30)$

\begin{tabular}{|c|c|c|c|c|c|c|c|c|c|}
\hline \multirow{2}{*}{ Group } & \multirow{2}{*}{ Baseline } & \multirow{2}{*}{ Intervention } & \multirow{2}{*}{ Follow-up } & \multirow{2}{*}{$\begin{array}{l}\text { Intervention } \\
\text { minus baseline }\end{array}$} & \multirow{2}{*}{$\begin{array}{l}\text { Follow-up } \\
\text { minus } \\
\text { intervention }\end{array}$} & \multicolumn{2}{|c|}{$\begin{array}{c}\text { Between } \\
\text { baseline and } \\
\text { intervention }\end{array}$} & \multicolumn{2}{|c|}{$\begin{array}{c}\text { Between } \\
\text { intervention } \\
\text { and follow-up }\end{array}$} \\
\hline & & & & & & $\begin{array}{c}p \text { - } \\
\text { value }\end{array}$ & $\begin{array}{c}\text { Cohen's } \\
\text { d }\end{array}$ & $\begin{array}{c}p- \\
\text { value }\end{array}$ & $\begin{array}{c}\text { Cohen's } \\
\text { d }\end{array}$ \\
\hline VG (n=15) & $31.92(23.08)$ & $26.45(16.32)$ & $27.48(11.42)$ & $-5.47(17.60)$ & $1.02(11.88)$ & 0.248 & 0.273 & 0.743 & 0.072 \\
\hline $\operatorname{VVG}(n=15)$ & $32.71(31.43)$ & $50.03(37.21)$ & $41.60(26.21)$ & $17.31(38.32)$ & $-8.42(33.01)$ & 0.102 & 0.502 & 0.339 & 0.261 \\
\hline \multicolumn{10}{|c|}{ Between groups } \\
\hline $\mathrm{t}(p)$ & & & & $-2.093(0.050)$ & $1.044(0.311)$ & & & & \\
\hline Cohen's d & & & & 0.764 & 0.381 & & & & \\
\hline
\end{tabular}

Values are presented as mean (SD).

VG: verbal feedback group, VVG: visual and verbal feedback group. 
aphragm is needed.

From this perspective, this study has significant implications for follow-up studies for retraining diaphragmatic contractions in a variety of patients, such as patients with LBP, sacroiliac joint pain, and stroke. It can also be proposed as a rehabilitation strategy that can activate and enhance diaphragmatic contraction as a component of core stability enhancement. Van et al. [11] showed that biofeedback using RUSI in healthy subjects is effective for lumbar multifidus muscle contraction, which is consistent with the results of this study. A total of 25 healthy volunteers were divided into 2 groups and received different types of biofeedback. Subjects were commonly subjected to verbal feedback on the amount of multifidus contraction, and only one group received additional visual biofeedback using RUSI during the acquisition phase. All subjects were reassessed a week later. Both groups showed significant improvement in the voluntary contraction of the multifidus muscle during the acquisition phase $(p<0.001)$, but the group with additional visual biofeedback showed greater improvement than the group with verbal feedback only $(p<0.05)$. In addition, the group that received visual ultrasound biofeedback retained their improvement in performance from week 1 to week $2(p<0.90)$, whereas the performance of the other group decreased $(p<0.05)$. In the results of this study, only the group that received visual and verbal feedback showed significant DT changes during the intervention session (medium effect size). However, this group did not continue to be effective during the follow-up session. This demonstrates that the verbal feedback provided in breathing interventions is more effective when additional visual feedback is applied. Diaphragm, one of the major inspiratory muscles, is more difficult to train than other trunk muscles due to the nature of the respiratory muscles and therefore requires special intervention, such as visual biofeedback.

The principles of motor learning explain how visual feedback improves diaphragm contraction. A methodology to contract these muscles is needed because of the low conscious sensory perception of the postural control muscles in the trunk and spine [18], including the diaphragm, where visual feedback plays a role. In the cognitive phase of motor learning proposed by Fitts and Posner [19], feedback, movement sequence, and instruction are required during repetitive exercises. At this phase, visual feedback provided using ultrasound images is most suitable. In addition, the visual feedback, which is provided in real time during muscle contraction, can provide information about the increased mus- cle thickness to improve the motor skill of activating the diaphragm. This knowledge can improve the motor skill required to shrink the diaphragm by informing the performer during task execution [20]. Ultrasound imaging has been used as a biofeedback tool for other muscles that contribute to spinal stability, including the transversus abdominis [13], lumbar multifidus [11], and pelvic floor [21]. Specifically, Richardson et al. [13] proposed ultrasound imaging as a biofeedback tool for neuromuscular re-education and found that ultrasound acts as a feedback to effectively activate trunk muscles in patients with LBP. In the same way that ultrasound imaging has been used in training the transversus abdominis, lumbar multifidus, and pelvic floor muscles, it may be a useful clinical tool for training individuals to engage the diaphragm to enhance spinal stability.

In conclusion, the use of the RUSI for visual feedback plus verbal feedback for instructing how to contract the diaphragm muscle in healthy subjects is a beneficial teaching tool for consistent performance of the diaphragm muscle compared to verbal feedback only, which is the teaching and feedback methods used in many clinics today. The effect of RUSI on the retention of diaphragm muscle performance was not found to be significant in this study. These results suggest that a protocol, such as the amount and intensity of the respiratory intervention method, is necessary to maintain the effect of the diaphragm breathing training continuously. The main limitation of this study is the relatively limited data acquisition methods. We used 2 physiotherapists skilled in ultrasound imaging to measure all subjects, which is not comparable to measurements made by inexperienced testers in ultrasound imaging. Further research is needed to determine if patients with diaphragm damage due to LBP or stroke would also benefit from the use of real-time ultrasound feedback in learning how to contact the diaphragm muscle. In future studies, it would be necessary to investigate whether patients with LBP or stroke benefit from relearning how to contract their diaphragm muscles in terms of pain reduction and functional improvements, such as respiratory performance.

\section{Conflict of Interest}

The authors declared no potential conflicts of interest with respect to the authorship and/or publication of this article. 


\section{References}

1. Harper CJ, Shahgholi L, Cieslak K, Hellyer NJ, Strommen JA, Boon AJ. Variability in diaphragm motion during normal breathing, assessed with B-mode ultrasound. J Orthop Sports Phys Ther 2013;43:927-31.

2. Hodges PW, Gandevia SC. Activation of the human diaphragm during a repetitive postural task. J Physiol 2000;522:165-75.

3. Hodges PW, Cresswell AG, Daggfeldt K, Thorstensson A. In vivo measurement of the effect of intra-abdominal pressure on the human spine. J Biomech 2001;34:347-53.

4. Hodges PW, Gandevia SC. Changes in intra-abdominal pressure during postural and respiratory activation of the human diaphragm. J Appl Physiol (1985) 2000;89:967-76.

5. Shirley D, Hodges PW, Eriksson AE, Gandevia SC. Spinal stiffness changes throughout the respiratory cycle. J Appl Physiol (1985) 2003;95:1467-75.

6. Kolar P, Sulc J, Kyncl M, Sanda J, Cakrt O, Andel R, et al. Postural function of the diaphragm in persons with and without chronic low back pain. J Orthop Sports Phys Ther 2012;42:35262.

7. O'Sullivan PB, Beales DJ, Beetham JA, Cripps J, Graf F, Lin IB, et al. Altered motor control strategies in subjects with sacroiliac joint pain during the active straight-leg-raise test. Spine (Phila Pa 1976) 2002;27:E1-8.

8. Cho JE, Lee HJ, Kim MK, Lee WH. The improvement in respiratory function by inspiratory muscle training is due to structural muscle changes in patients with stroke: a randomized controlled pilot trial. Top Stroke Rehabil 2017;25:37-43.

9. Zivkovic V, Lazovic M, Vlajkovic M, Slavkovic A, Dimitrijevic L, Stankovic I, et al. Diaphragmatic breathing exercises and pelvic floor retraining in children with dysfunctional voiding. Eur J Phys Rehabil Med 2012;48:413-21.

10. Cho JE, Cho KH, Yoo JS, Lee SJ, Lee WH. Reliability and val- idity of a dual-probe personal computer-based muscle viewer for measuring the pennation angle of the medial gastrocnemius muscle in patients who have had a stroke. Top Stroke Rehabil 2017;25:6-12.

11. Van K, Hides JA, Richardson CA. The use of real-time ultrasound imaging for biofeedback of lumbar multifidus muscle contraction in healthy subjects. J Orthop Sports Phys Ther 2006;36:920-5.

12. Henry SM, Westervelt KC. The use of real-time ultrasound feedback in teaching abdominal hollowing exercises to healthy subjects. J Orthop Sports Phys Ther 2005;35:338-45.

13. Richardson C, Hodges P, Hides J. Therapeutic exercise for lumbopelvic stabilization: a motor control approach for the treatment and prevention of low back pain. New York, NY: Churchill Livingstone; 2004.

14. Kim T, Pollock S, Lee D, O'Brien R, Keall P. Audiovisual biofeedback improves diaphragm motion reproducibility in MRI. Med Phys 2012;39:6921-8.

15. Cohen J. Statistical power analysis for the behavioral sciences. 2nd ed. Hillsdale: Erlbaum Associates; 1988.

16. Parkhurst TM, Burnett CN. Injury and proprioception in the lower back. J Orthop Sports Phys Ther 1994;19:282-95.

17. Oh D, Kim G, Lee W, Shin MM. Effects of inspiratory muscle training on balance ability and abdominal muscle thickness in chronic stroke patients. J Phys Ther Sci 2016;28:107-11.

18. Kandel ER, Schwartz JH, Jessell TM. Principles of neural science. 4th ed. New York, NY: McGraw-hill; 2000.

19. Fitts PM, Posner MI. Human performance. Belmont: Brooks/ Cole; 1967. p. 7-16.

20. Salmoni AW, Schmidt RA, Walter CB. Knowledge of results and motor learning: a review and critical reappraisal. Psychol Bull 1984;95:355-86.

21. Dietz HP. Ultrasound imaging of the pelvic floor. Part I: two-dimensional aspects. Ultrasound Obstet Gynecol 2004;23:80-92. 